Noname manuscript No.

(will be inserted by the editor)

\title{
Alattin: Mining Alternative Patterns for Defect Detection
}

\author{
Suresh Thummalapenta - Tao Xie
}

the date of receipt and acceptance should be inserted later

\begin{abstract}
To improve software quality, static or dynamic defect-detection tools accept programming rules as input and detect their violations in software as defects. As these programming rules are often not well documented in practice, previous work developed various approaches that mine programming rules as frequent patterns from program source code. Then these approaches use static or dynamic defect-detection techniques to detect pattern violations in source code under analysis. However, these existing approaches often produce many false positives due to various factors. To reduce false positives produced by these mining approaches, we develop a novel approach, called Alattin, that includes new mining algorithms and a technique for detecting neglected conditions based on our mining algorithm. Our new mining algorithms mine patterns in four pattern formats: conjunctive, disjunctive, exclusive-disjunctive, and combinations of these patterns. We show the benefits and limitations of these four pattern formats with respect to false positives
\end{abstract}

This paper is an extended version of our previous work published at ASE 2009 [Thummalapenta and Xie(2009a)]. Our previous work introduced the concept of balanced and imbalanced patterns that are expressed in the Or pattern format. In this work, we propose additional new pattern formats Xor and Combo. We also propose new mining algorithms for mining patterns in $O r, X o r$, and Combo pattern formats. Furthermore, we show the benefits and limitations of And, Or, Xor, and Combo pattern formats by applying the patterns mined using these formats to the problem of detecting neglected conditions in applications under analysis.

Suresh Thummalapenta

Department of Computer Science

North Carolina State University

Raleigh

E-mail: sthumma@ncsu.edu

Tao Xie

Department of Computer Science

North Carolina State University

Raleigh

E-mail: xie@csc.ncsu.edu 
and false negatives among detected violations by applying those patterns to the problem of detecting neglected conditions.

\section{Introduction}

Programming rules serve as a basis for applying static or dynamic defectdetection tools to detect rule violations as software defects and improve software quality. However, in practice, these programming rules are often not well documented for Application Programming Interfaces (APIs) due to various factors such as hard project delivery deadlines and limited resources in the software development process [Lethbridge et al(2003)Lethbridge, Singer, and Forward]. To tackle the issue of lacking documented programming rules, various approaches have been developed in the past decade to mine programming rules from program executions [Ernst et al(2001)Ernst, Cockrell, Griswold, and Notkin, Ammons et al(2002)Ammons, Bodik, and Larus, Yang et al(2006)Yang, Evans, Bhardwaj, Bhat, and Das], individual versions [Engler et al(2001)Engler, Chen, Hallem, Chou, and Chelf,Li and Zhou(2005), Acharya et al(2006)Acharya, Xie, and Xu,Ramanathan et al(2007)Ramanathan, Grama, and Jagannathan,Shoham et al(2007)Shoham, Yahav, Fink, and Pistoia,Chang et al(2007)Chang, Podgurski, and Yang, Acharya et al(2007)Acharya, Xie, Pei, and $\mathrm{Xu}$, Wasylkowski et al(2007)Wasylkowski, Zeller, and Lindig], or version histories [Livshits and Zimmermann(2005),Williams and Hollingsworth(2005)] of program source code. A methodology, commonly referred to as mining software engineering data (MSED) [DMSE(2010)], adopted by these approaches is to mine common patterns (e.g., frequent occurrences of pairs or sequences of API calls) across a sufficiently large number of data points (e.g., code examples). These common patterns often reflect programming rules that should be obeyed when programmers write code using API calls involved in these rules. Then, these approaches use static or dynamic defect-detection techniques that accept mined patterns as inputs and detect pattern violations as potential defects in applications under analysis.

Since the inception of the MSED methodology, the major focus is to mine various types of patterns and use those patterns for detecting defects in applications under analysis. Although MSED has been shown to be effective in detecting defects in applications under analysis, a major drawback of MSED is that the violations detected by existing MSED-based approaches often include a large number of false positives. This phenomenon is reflected in the empirical evaluations of existing approaches [Engler et al(2001)Engler, Chen, Hallem, Chou, and Chelf, Li and Zhou(2005), Acharya et al(2006)Acharya, $\mathrm{Xie}$, and $\mathrm{Xu}$, Chang et al(2007)Chang, Podgurski, and Yang, Wasylkowski et al(2007)Wasylkowski, Zeller, and Lindig] where majority of detected violations turn out to be false positives.

To illustrate how detected violations turn out to be false positives, we use two code examples (shown in Figure 1) using the next method of the Iterator class. The next method throws NoSuchElementException when invoked on an 


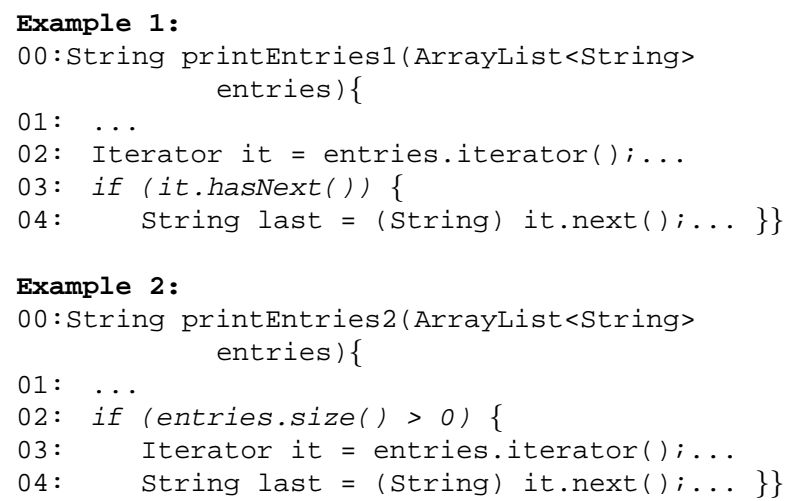

Fig. 1 Two code examples using the next method of the Iterator class

ArrayList object without any elements. Programmers can avoid this exception by using either the condition check " $P_{1}$ : boolean-check on return of Iterator.hasNext before Iterator. next" (shown in printEntries 1 from Example 1) or " $P_{2}$ : const-check on return of ArrayList.size before Iterator.next" (shown in printentries2 from Example 2). In general, programmers use either $P_{1}$ or $P_{2}$ but not both, since using both $P_{1}$ and $P_{2}$ is redundant. Consider that a single pattern $P_{1}$ is mined from the data points. A static defect-detection technique reports a violation in printentries2, since the method does not satisfy $P_{1}$. However, the code example does not include any defect on using Iterator.next, since printEntries 2 satisfies $P_{2}$; therefore, the detected violation turns out to be a false positive.

In our empirical investigation of false positives generated by existing approaches [Engler et al(2001)Engler, Chen, Hallem, Chou, and Chelf, Li and Zhou(2005),Acharya et al(2006)Acharya, Xie, and Xu,Chang et al(2007)Chang, Podgurski, and Yang, Wasylkowski et al(2007)Wasylkowski, Zeller, and Lindig], we identify that a major reason for such a large number of false positives is that the focus of MSED is to mine single patterns ( $\operatorname{such}$ as $P_{1}$ ) or conjunctive patterns (such as $P_{1} \wedge P_{2}$ ) (more details are presented in Section 5). The conjunctive pattern $P_{1} \wedge P_{2}$ describes that both $P_{1}$ and $P_{2}$ often appear together among the data points (e.g., code examples). We identify that these single or conjunctive patterns alone cannot describe the nearly complete behavior among data points, resulting in false positives. The reason why single or conjunctive patterns are not sufficient is that programmers write source code in different ways to achieve the same programming task (as shown in Examples 1 and 2). For example, the pattern $P_{1} \oplus P_{2}{ }^{1}$ describes both the condition checks that can be used before the next method. Furthermore, using the pattern $P_{1} \oplus P_{2}$ does not result in violations in printentries 1 and printentries2, thereby reducing false positives. We focus on mining patterns that describe nearly complete rather than complete behavior, since the patterns that describe complete behavior cannot help detect violations as deviations from those patterns, resulting in false negatives.

1 The symbol $\oplus$ represents the exclusive-or relationship. 
To reduce both false positives and false negatives among detected violations and to infer patterns that describe nearly complete behavior, in this paper, we propose a novel approach, called Alattin, that includes new mining algorithms and a technique that detects neglected conditions (described next) using patterns mined by our mining algorithms. In particular, our algorithms mine patterns in four pattern formats: conjunctive $(A n d$ or $\wedge)$, disjunctive $(O r$ or $\vee$ ), exclusive-disjunctive (Xor or $\oplus$ ), and combinations of these patterns (referred to as Combo patterns). We use Alternative Patterns to collectively refer to patterns of all four formats and refer to individual patterns such as $P_{1}$ and $P_{2}$ in $P_{1} \wedge P_{2}$ as alternatives.

In general, mining $O r$ and $X o r$ patterns is more challenging than mining And patterns, since $O r$ and Xor patterns do not follow the Apriori principle [Han and Kamber(2000)] in data mining. Given an input database of itemsets for applying mining techniques, the Apriori principle states that if an itemset is frequent, then all its subsets should also be frequent. Existing mining techniques [Burdick et al(2001)Burdick, Calimlim, and Gehrke] that target at mining And patterns use this principle for pruning the search space. For example, if an itemset $P_{1}$ is not frequent, then any super $A n d$ itemset of $P_{1}$ such as $P_{1} \wedge P_{2}$ cannot be frequent, and hence can be pruned. However, the Apriori principle does not hold for mining $O r$ and $X o r$ patterns. For example, although the itemset $P_{1}$ is not frequent, its super $O r$ itemset such as $P_{1} \vee P_{2}$ can be frequent, since $P_{1} \vee P_{2}$ is supported by more itemsets in the input database compared to $P_{1}$ or $P_{2}$ individually.

In this paper, we show the benefits and limitations of these four pattern formats with respect to false positives and false negatives by applying these pattern formats to the problem of detecting neglected conditions. Neglected conditions, also referred to as missing paths, are known to be an important category of software defects and are considered to be one of the primary reasons for many fatal issues such as security or buffer overflow vulnerabilities [Chang et al(2007)Chang, Podgurski, and Yang]. As shown by a recent study [Chang et al(2007)Chang, Podgurski, and Yang], 66\% (109/167) of defect fixes applied in the Mozilla Firefox project are due to neglected conditions. In particular, neglected conditions (related to an API call) refer to (1) missing conditions that check the arguments or receiver of the API call before the API call or (2) missing conditions that check the return values or receiver of the API call after the API call. In our approach, we mine patterns that describe necessary condition checks related to an API call in these four formats and use those condition checks for detecting neglected conditions in applications under analysis.

This paper makes the following main contributions:

- An empirical investigation of four pattern formats: conjunctive $($ And or $\wedge)$, disjunctive $(O r$ or $\vee$ ), exclusive-disjunctive (Xor or $\oplus$ ), and combinations of these patterns (referred to as combo patterns) in software engineering data.

- New mining algorithms for efficiently mining patterns in Or, Xor, and Combo pattern formats. 


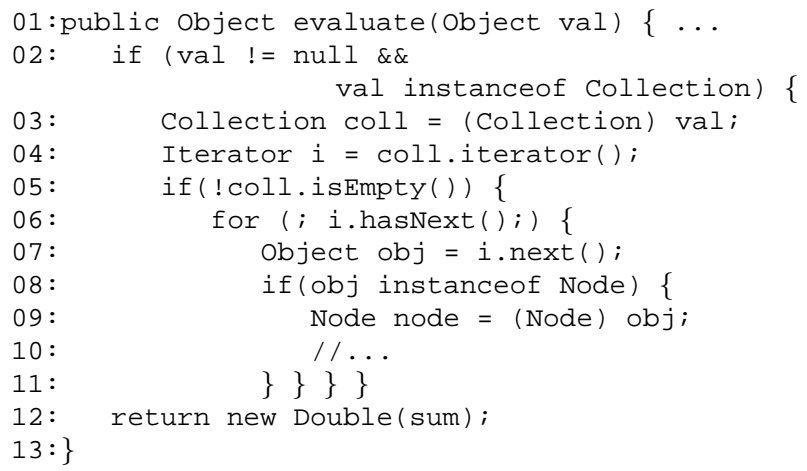

Fig. 2 A code example using Iterator. next gathered from Google code search.

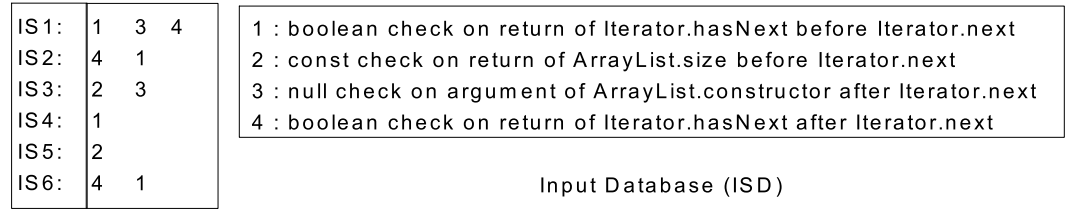

Fig. 3 An example input database $I S D$.

- A technique that applies patterns of these four pattern formats for detecting neglected conditions around individual API calls in an application under analysis.

- Two evaluations to demonstrate the effectiveness of our approach. Our evaluation results show that the best pattern-mining approach (in terms of reducing both false positives and false negatives) is to first mine And patterns for API methods and next mine Combo patterns. Among violations detected by Combo patterns, the best violation-detection approach is to assign higher priority to the violations of API methods with And patterns compared to the violations of API methods without And patterns. The primary reason is that the former violations are more likely to be real defects compared to the latter violations.

\section{Example}

We next use an illustrative example to describe our approach on how we collect the data that describes necessary condition checks around API calls. We also show how our proposed four pattern formats affect the number of false negatives and false positives among detected violations. Consider that an application under analysis uses the Iterator. next method as shown in the printEntries2 method in Figure 1.

Initially, we gather relevant code examples that invoke the Iterator.next method by constructing queries to Google code search [GCSE(2006)]. These relevant code examples are required for mining patterns that describe nec- 
essary condition checks around the Iterator.next method. Figure 2 shows a code example gathered from Google code search. We next construct controlflow graphs (CFG) for collected code examples and perform two traversals (backward and forward) of the CFG from the node corresponding to the Iterator.next method. In the backward traversal, we collect the condition checks on the receiver and argument objects preceding the call site of the Iterator.next method. Similarly, in the forward traversal, we collect the condition checks on the receiver and return objects succeeding the call site of the Iterator.next method. For the current code example, our backward and forward traversals gather the following condition checks.

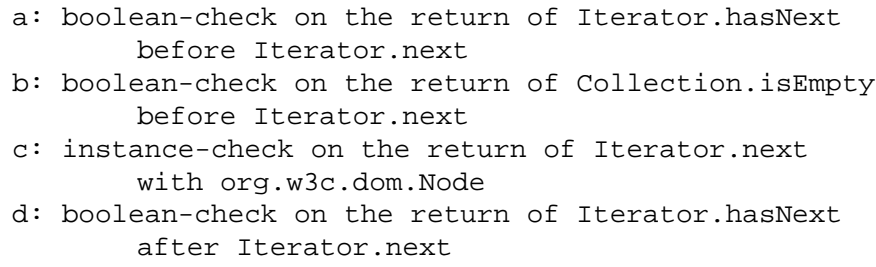

Condition check "a" describes the condition check performed before the call site of the next method, whereas Condition check "d" describes the condition check performed after the call site of the next method. The reason for two condition checks is that the next method (Statement 7) is invoked in a for loop. Section 4 presents more details on how we exploit program dependencies while performing backward and forward traversals. The preceding set of condition checks collected from the code example forms an itemset in the itemset database $I S D$, used as input for mining patterns. We analyze all gathered code examples to generate various itemsets and use different mining algorithms for mining the patterns in formats: And, Or, Xor, and Combo. Section 3 presents more details on our mining algorithms.

Figure 3 shows a sample itemset database $I S D$ with six itemsets. This $I S D$ includes four distinct items labeled with IDs 1 through 4 . The figure also shows the condition check corresponding to each item. We next apply our mining algorithms for mining different pattern formats. The patterns mined by our algorithms with a minimum support threshold value min_sup of 0.4 are shown below:

- And Pattern: "1 14 ", support: 0.5

- Or Pattern: "1 $12 \vee 3 \vee 4$ ", support: 1.0

- Xor Pattern: "1 $\oplus 2$ ", support 1.0; "4", support: 0.5

- Combo Pattern: " $(1 \wedge 4) \oplus 2$ ", support: 0.83

Among the itemsets shown in ISD, Items 1 and 4 often appear together with an $\wedge$ relation, since the methods hasNext and next are often used in a loop (as shown in Statements 6 and 7 in Figure 2). Although the And pattern captures this behavior, the And pattern cannot capture the relation with Item 2, resulting in false positives when applied on code examples such as printentries2 in Figure 1. On the other hand, the Or pattern does not result 
in false positives, since the pattern includes all items. However, the $O r$ pattern does not help in detecting violations, resulting in false negatives. Although the Xor pattern can perform better than the Or pattern, the Xor pattern may not detect violations in code examples, which include only Item 1 and do not include Item 4. As shown in this example, the Combo pattern describes the nearly complete behavior and also helps reduce both false positives and false negatives.

Along with the challenges faced while mining Or and Xor patterns, Combo patterns pose additional challenges in choosing the suitable operator while combining items. For example, the suitable operator for combining items "1" and " 4 " is $\wedge$, although " $1 \vee 4$ " results in a higher support value than " $1 \wedge 4$ ". We describe these challenges in subsequent sections and present our algorithms for mining these patterns.

In summary, this example illustrates the existence of pattern formats And, Or, and Xor, and also shows that no single pattern format alone can help in describing the necessary condition checks around API calls.

\section{Mining Algorithms for Alternative Patterns}

We next describe four pattern formats that we use in our empirical study. We first present formal definitions for the four pattern formats and next describe our algorithms for mining the pattern in four formats with illustrative examples.

\subsection{Formal Definitions}

Let $M=\left\{m_{1}, m_{2}, \ldots, m_{k}\right\}$ be the set of all possible distinct items. For example, an $m_{j}$ represents a condition check such as "boolean-check on return of Iterator. hasNext before Iterator. next". Consider an ItemSet Database ISD as $\left\{i s_{1}, i s_{2}, \ldots, i s_{l}\right\}$, where each itemset $i s_{j}$ includes different sets of elements such as $\left\{m_{1}, m_{2}, \ldots, m_{a}\right\}$ from the set of all possible distinct elements.

Definition 1 Pattern Candidate. A pattern candidate $p c$ is a single item $m_{k} \in M$ or a combination of two elements associated by a logical operator $o p \in\{\wedge, \vee, \oplus\}$. Each element in the combination is an item $m_{i} \in M$ or another pattern candidate.

The preceding definition is a recursive definition, which defines that a pattern candidate can be either a simple or nested pattern candidate. For example, a simple pattern candidate $p c_{k}: m_{i} \wedge m_{j}$ is a combination of two items $m_{i}$ and $m_{j}$ with the operator $o p \in\{\wedge\}$. On the other hand, a nested pattern candidate $p c_{l}: m_{i} \vee p c_{k}$ is a combination of an item $m_{i}$ and the preceding pattern candidate $p c_{k}$ with the operator $o p \in\{\mathrm{V}\}$. We use notations $p c_{k}$.left and $p c_{k}$.right to refer to the left and right child pattern candidates, respectively, and refer to $p c_{k}$ as their parent pattern candidate. Furthermore, we use the 
notation $p c_{k} . o p$ to refer to the operator op of the pattern candidate $p c_{k}$. We classify a pattern candidate by its format, especially using its operator.

Definition 2 And Pattern Candidate. An And pattern candidate is a pattern candidate where the operator op $\in\{\wedge\}$ and all the child pattern candidates are also And pattern candidates.

Definition 3 Or Pattern Candidate. An Or pattern candidate is a pattern candidate where the operator $o p \in\{\bigvee\}$ and all the child pattern candidates are also Or pattern candidates.

Definition 4 Xor Pattern Candidate. A Xor pattern candidate is a pattern candidate where the operator $o p \in\{\oplus\}$ and all the child pattern candidates are also Xor pattern candidates.

Definition 5 Combo Pattern Candidate. A Combo pattern candidate is a pattern candidate where the operator op $\in\{\wedge, \vee, \oplus\}$.

The category of Combo pattern candidates subsumes the categories of $A n d$, Or, and Xor pattern candidates. An example combo pattern candidate is " $p c_{1} \oplus p c_{2}$ ", where $p c_{1}$ and $p c_{2}$ are " $m_{i} \wedge m_{j}$ " and " $m_{k} \wedge m_{l}$ ", respectively.

To compute frequent patterns among pattern candidates, we use a threshold value, referred to as min_sup, that describes the minimum support for a pattern candidate to be classified as a frequent pattern. Algorithm 1, IssupportedBy, describes how we compute support values for the preceding pattern formats. In particular, Is SupportedBy accepts a pattern candidate $p c_{k}$ and an itemset $i s_{j}$, and returns true, if $i s_{j}$ supports $p c_{k}$, and otherwise returns false. Initially, Is SupportedBy checks whether $p c_{k}$ is a single item and returns true or false based on whether $p c_{k}$ is contained in $i s_{j}$. Otherwise, Is SupportedBy recursively computes whether $p c_{k}$.left and $p c_{k}$.right of $p c_{k}$ are supported by the itemset $i s_{j}$, and uses the operator $p c_{k}$.op for checking whether $i s_{j}$ supports $p c_{k}$ (Lines 4 - 15). We compute the support value of a pattern candidate $p c_{k}$, referred to as support $\left(p c_{k}\right)$, based on the number of the itemsets (in $I S D$ ) that return true for the algorithm IssupportedBy.

Definition 6 Frequent Pattern (FP). A pattern candidate $p c_{k}$ is considered as a frequent pattern, if $\operatorname{support}\left(p c_{k}\right) \geq \operatorname{min\_ sup.}$

A frequent pattern $f p_{k}$ is considered as an And, Or, Xor, or Combo pattern based on the operator $f p_{k} . o p$.

\subsection{Mining Algorithms}

We next present our algorithms for mining preceding pattern formats. In particular, we present algorithms for mining Or, Xor, and Combo patterns, since mining And patterns can be achieved by well-known approaches such as a frequent itemset miner [Burdick et al(2001)Burdick, Calimlim, and Gehrke]. We first explain our algorithm for mining $O r$ and Xor patterns, and next describe the algorithm for mining Combo patterns. 


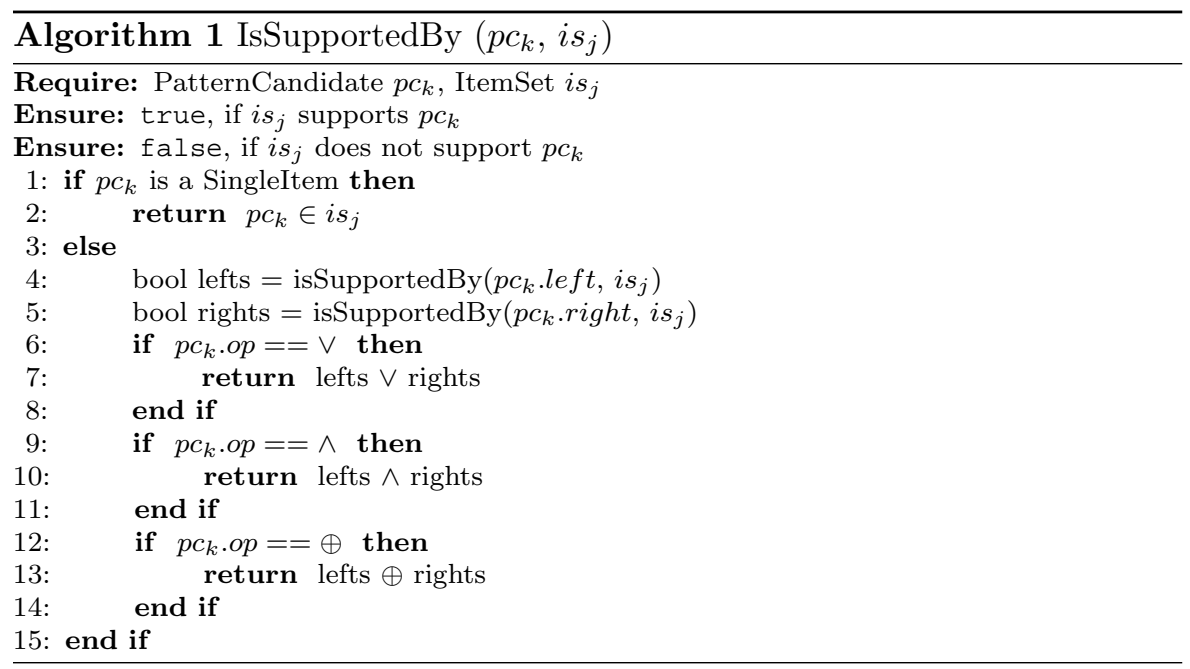

\subsubsection{Mining Or and Xor Patterns}

In general, mining $O r$ and Xor patterns is more challenging than mining And patterns, since these patterns do not follow the Apriori principle [Han and Kamber(2000)]. The Apriori principle states that if an itemset is frequent, then all its subsets should also be frequent. Existing mining techniques that target at mining And patterns use this principle for pruning the search space. For example, if an itemset $p c_{1}$ is not frequent, then any super itemset of $p c_{1}$ such as $p c_{1} \wedge p c_{2}$ cannot be frequent, and hence can be pruned. However, the Apriori principle does not hold for mining $O r$ and Xor patterns. For example, even if the itemset $p c_{1}$ is not frequent, then its super itemset such as $p c_{1} \vee p c_{2}$ can be frequent. To address this issue, we propose a greedy algorithm based on the following property for pruning the search space of patterns. This property is inspired by Nanavati et al. [Nanavati et al(2001)Nanavati, Chitrapura, Joshi, and Krishnapuram] and is applicable to both $O r$ and Xor patterns.

Property 1 The support of an $O r$ or Xor pattern candidate $p c_{k}$, represented as support $\left(p c_{k}\right)$, formed from two pattern candidates $p c_{k}$.left and $p c_{k}$.right should have higher value than support ( $p c_{k} . l e f t$ ) and support ( $\left.p c_{k} . r i g h t\right)$.

The rationale behind the preceding property is based on our objective to mine patterns that describe nearly complete behavior. For example, a pattern candidate $p c_{k}=p c_{i} \vee p c_{j}$, whose support value is less than support ( $p c_{i}$ ) and support $\left(p c_{j}\right)$ is not useful compared to individual pattern candidates in achieving our objective. Algorithm 2, Minexoror, describes our greedy algorithm for mining $O r$ and Xor patterns. MineXoror accepts itemset database $I S D$, min_sup, and ptype $\in\{\vee, \oplus\}$ as inputs.

Initially, MineXoror identifies all distinct items in the itemset database $I S D$ using the function ComputeDistinct (Line 2). Among these distinct items, 


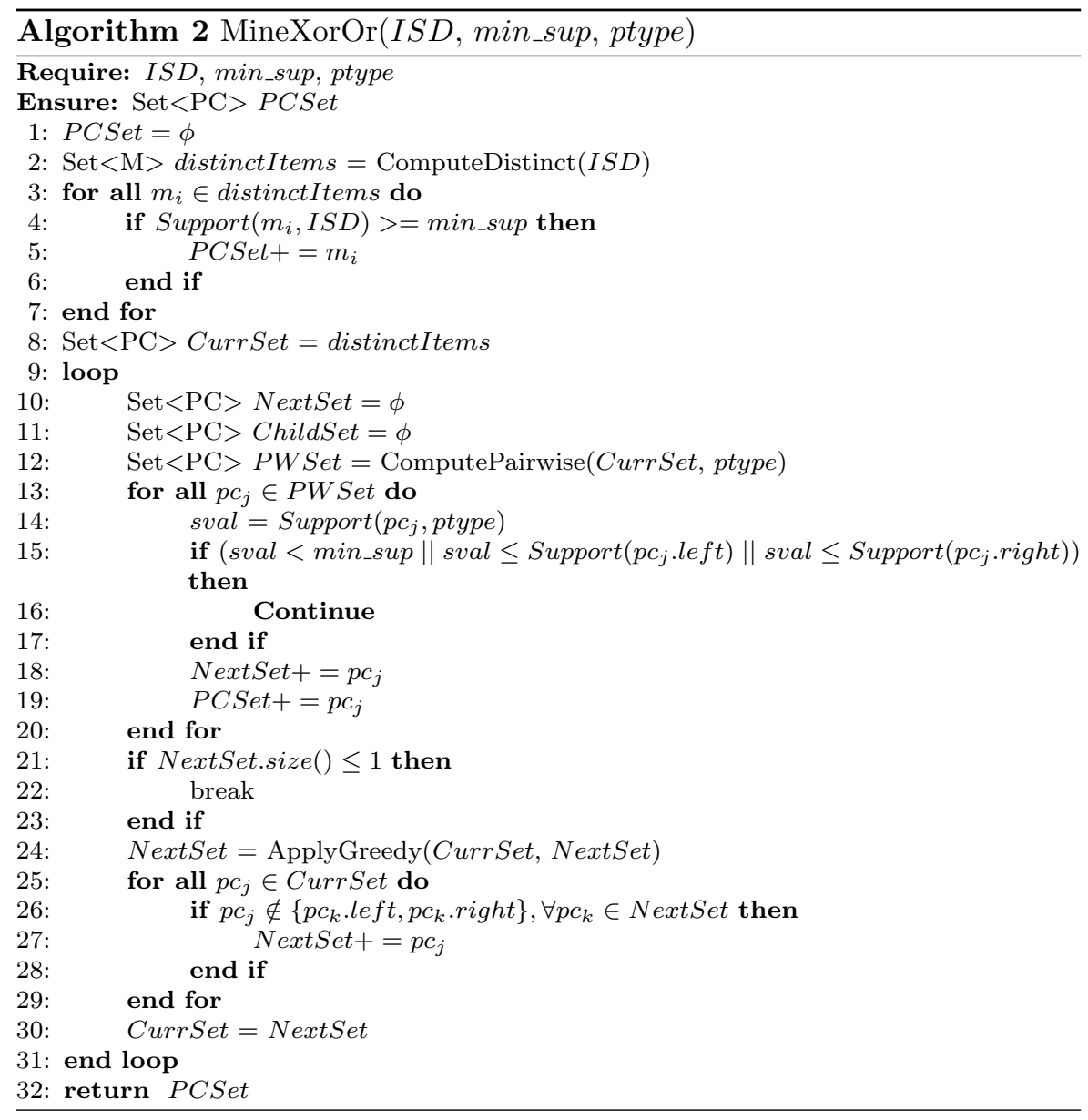

Minexoror checks whether the support of any of these items is greater than min_sup and adds those items to PCSet (Lines 3 to 7). Next, Minexoror uses various iterations, where pairwise combinations are computed using the function computepairwise from the elements of the previous iteration stored in CurrSet (Lines 9 to 31). For example, consider Or patterns. For the currset $=\left\{p c_{1}, p c_{2}, p c_{3}\right\}$, ComputePairwise returns a set with three elements, i.e., Pwset $=\left\{p c_{1} \vee p c_{2}, p c_{1} \vee p c_{3}, p c_{2} \vee p c_{3}\right\}$. The algorithm next identifies the elements in PwSet, whose support values are greater than min_sup and satisfy Property 1. Minexoror next chooses pattern candidates (from NextSet) that participate in the next iteration by greedily choosing one parent pattern candidate in NextSet with the highest support value for each pattern candidate in CurrSet using the function ApplyGreedy (Line 24). The rationale behind our greedy approach is based on our empirical investigation that the real patterns describing necessary condition checks around API calls often have higher support values compared to other patterns. Finally, Minexoror identifies those pattern candi- 


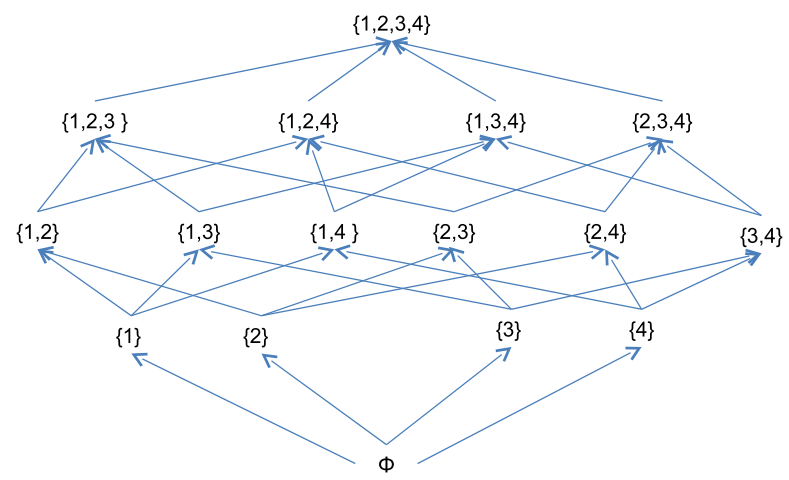

Fig. 4 All possible itemsets with four distinct items.

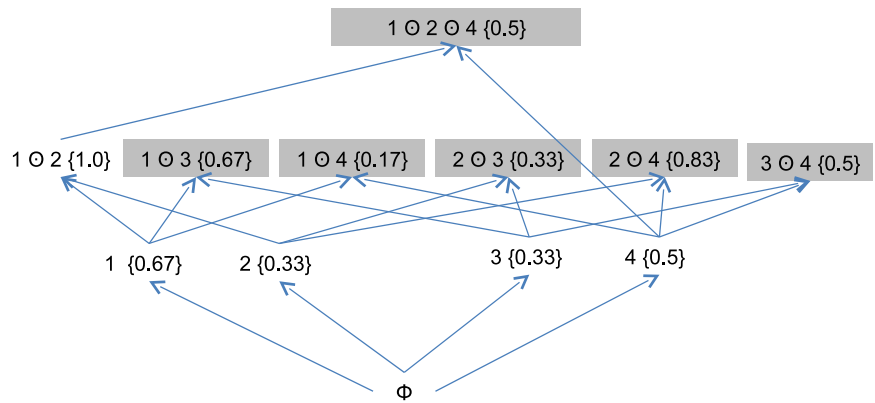

Fig. 5 Mining Xor patterns.

dates in CurrSet whose parent pattern candidates do not belong to NextSet (Lines 25 - 29). MineXoror adds such pattern candidates to NextSet as well, since these pattern candidates can still be helpful when combined with other pattern candidates in NextSet.

We next explain the algorithm in detail using the itemset database shown in Figure 3. The itemset database includes four distinct items. Figure 4 shows all possible pattern candidates that can be derived using the preceding four distinct items. This figure shows the complete search space of pattern candidates. We first explain mining Xor patterns and next explain mining $O r$ patterns.

Mining Xor Patterns. Figure 5 shows how Minexoror prunes the search space and generates patterns " $1 \oplus 2$ " (support 1.0) and " 4 " (support: 0.5). The value shown in braces next to each pattern candidate indicates support value of that pattern candidate. The pattern candidates shown in gray are pruned by MineXoror due to three factors. First, the support value of pattern candidate is lower than min_sup. For example, support $(2 \oplus 3)=0.33$, which is lower than min_sup. Second, the pattern candidate does not satisfy Property 1. For example, the pattern candidate " $1 \oplus 3$ " does not satisfy Property 1 , since support $(1 \oplus 3) \leq \operatorname{support}(1)$. Third, the pattern candidate is not the candidate with the highest support value among the parent pattern candidates of each 


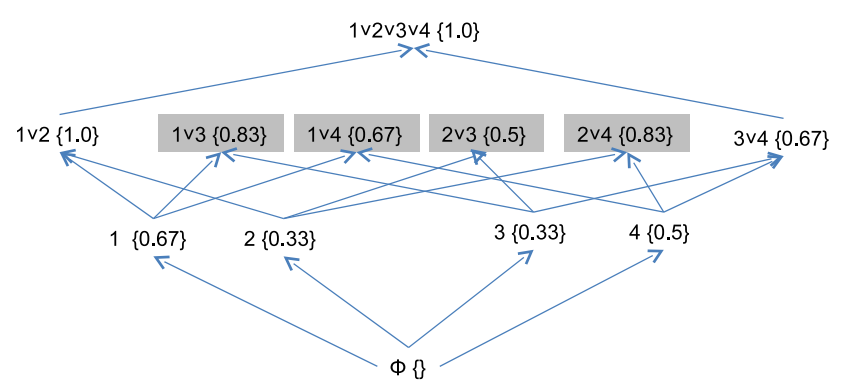

Fig. 6 Mining Or patterns.

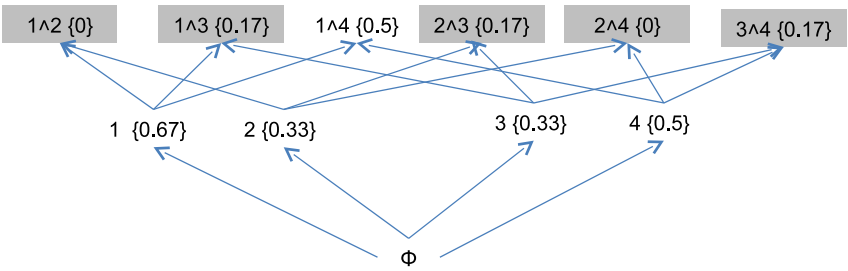

Fig. 7 Phase 1 of mining Combo patterns.

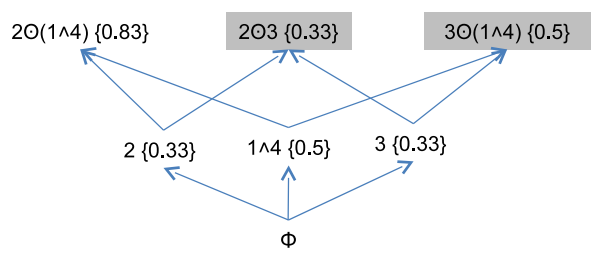

Fig. 8 Phase 2 of mining Combo patterns.

child pattern candidate. For example, the pattern candidate " $2 \oplus 4$ " is pruned, since the pattern candidate " $1 \oplus 2$ " has a higher support value than " $2 \oplus 4$ ". Since all parent pattern candidates of "4" are pruned away, MineXoror adds this candidate to NextSet for the next iteration and computes further pattern candidates such as " $1 \oplus 2 \oplus 4$ ".

Mining Or Patterns. Figure 6 shows how Minexoror prunes the search space and generates the $O r$ pattern " $1 \vee 2 \vee 3 \vee 4$ " (support: 1.0). Similar to Xor patterns, the pattern candidates are pruned due to the same three factors.

\subsubsection{Mining Combo Patterns}

We next describe how we mine combo patterns. The algorithm for mining Combo patterns includes two phases. Phase 1 mines And Patterns and Phase 2 mines Combo patterns using the output of Phase 1. We next explain each phase in detail.

Phase 1. Algorithm 3, MineCombop1, shows Phase 1 of mining Combo patterns. In particular, MineComboP1 computes pairwise And combinations of all pattern candidates in CurrSet and checks whether new pattern candidates 


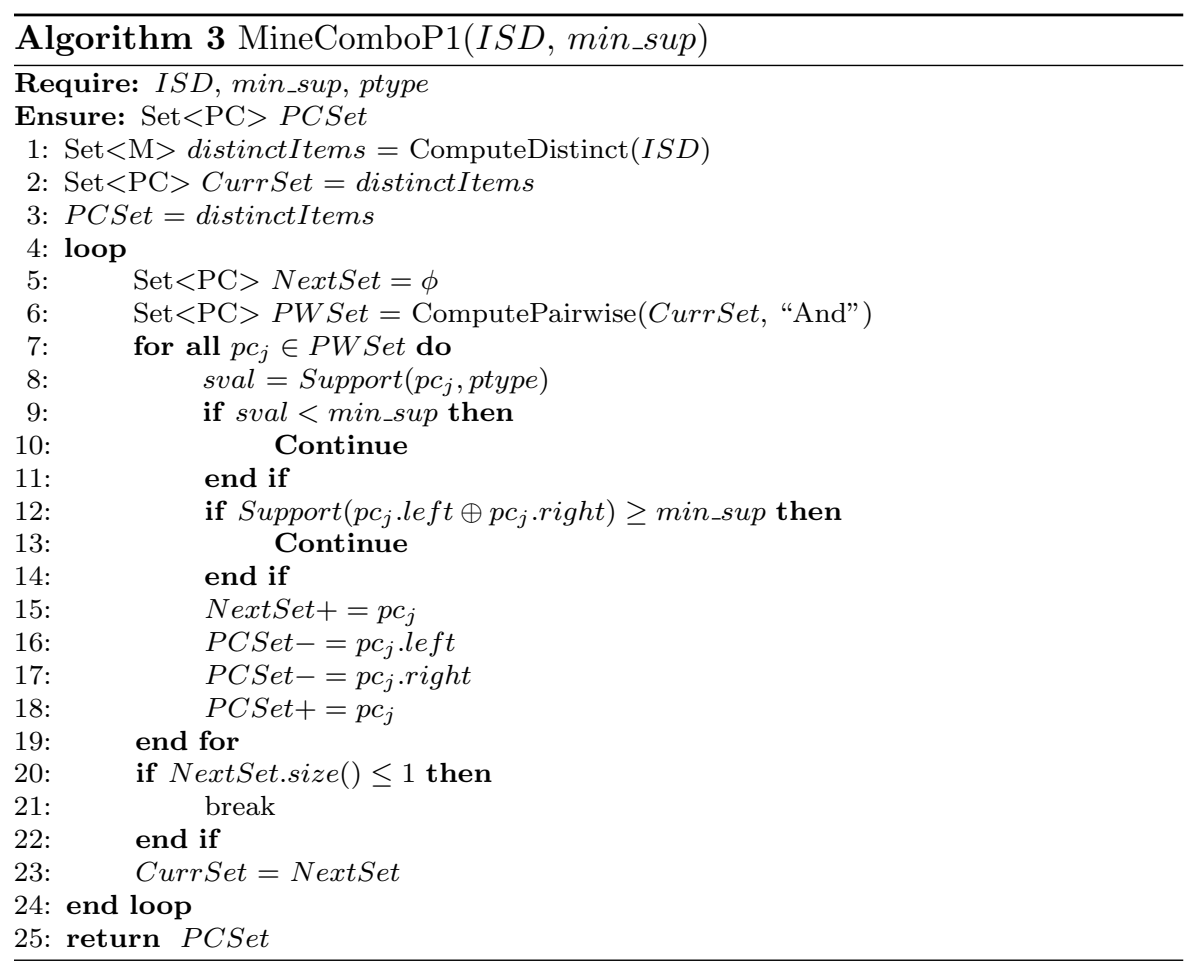

have higher support values than min_sup (Lines 9 - 11). If yes, MineCombop1 computes the support of Xor combination of the two candidates, shown as Support $\left(p c_{j}\right.$. left $\oplus p c_{j}$.right $)$ in Line 12. If the preceding support value is also higher than min_sup, then MineComboP1 ignores the And combination. The rationale behind this decision is that, if both " $p c_{j} . l e f t \wedge p c_{j} . r i g h t "$ and " $p c_{j}$.left $\oplus p c_{j}$.right" have higher values than min_sup, then the suitable combination of $p c_{j}$.left and $p c_{j}$. right is " $p c_{j}$.left $\vee p c_{j} . r i g h t$ ".

Figure 7 shows the output of MineCombor 1 with the itemset database ISD shown in Figure 3. As shown in the figure, Phase 1 produces three pattern candidates as output: "1 14 ", "2", and "3", which are passed as inputs to Phase 2.

Phase 2. Phase 2 of mining Combo patterns is similar to Algorithm 2 for mining Xor and Or patterns. The major difference is to choosing a suitable operator, op $\in\{\vee, \oplus\}$, when combining two pattern candidates during the computation of pairwise combinations. Given two pattern candidates $p c_{i}$ and $p c_{j}$,

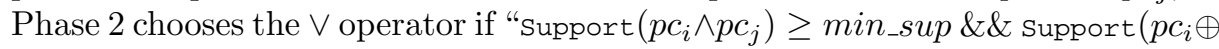

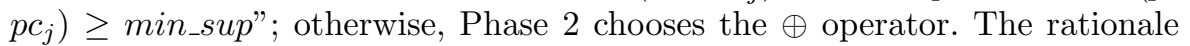
behind this decision is the same as the reason given in Phase 1. Figure 8 shows the output of Phase 2 resulting in the pattern " $(1 \wedge 4) \oplus 2$ " (support: 0.83 ). 


\section{Alattin Approach}

Our Alattin approach accepts an application under analysis and detects neglected conditions around APIs reused by the application. More specifically, Alattin scans the application and gathers APIs reused by the application. Alattin uses our mining algorithms to mine patterns that serve as programming rules in reusing those APIs. Then Alattin detects violations of these programming rules. In summary, Alattin includes four major phases. In Phase 1, Alattin gathers relevant code examples that reuse APIs. In Phase 2, Alattin analyzes gathered code examples or application under analysis to generate pattern candidates suitable for mining. In Phase 3, Alattin applies mining algorithms on pattern candidates to mine patterns. In Phase 4 , Alattin detects violations of mined patterns in the application under analysis. We next explain each phase in detail. We use notations $C_{i}$ and $F_{i}$ to denote a class or a method used by the application under analysis, respectively.

\subsection{Phase 1: Gathering Code Examples}

In Phase 1, Alattin gathers code examples from existing open source repositories through code search engines (CSE) such as Google code search [GCSE(2006)] and Koders [KODERS(2005)]. These code search engines are primarily used by programmers in searching for relevant code examples from available open source projects on the web. As these CSEs can serve as powerful resources of open source code, these CSEs can be exploited for other tasks such as detecting violations in applications that reuse existing open source projects. Our approach uses a CSE to gather relevant code examples and mines gathered code examples to detect violations in an application under analysis.

To collect code examples through a CSE, Alattin constructs queries for each $C_{i}$ and $F_{i}$. For example, Alattin constructs query of the form "lang: java Iterator next" to collect code examples that invoke the next method of the Iterator class. More specifically, our queries include the names of the class and method along with the language type. Alattin stores gathered code examples in the local file system for further analysis. Alattin uses Google code search (GCSE) [GCSE(2006)] for collecting relevant code examples with two main reasons: (1) GCSE provides client libraries that can be used by other tools to interact with and (2) GCSE has public forums that provide good support. However, our approach is independent of GCSE and can leverage any other CSE to gather relevant code examples.

\subsection{Phase 2: Generating Pattern Candidates}

In Phase 2, Alattin analyzes gathered code examples or application under analysis statically to generate pattern candidates suitable for mining. These pattern candidates include condition checks that are performed before and after invoking an $F_{i}$ method. To identify these condition checks on method calls, 
Alattin has to associate condition checks in the conditional expressions of If or while statements with the related method calls. We use the Iterator.next method and its relevant code example in Figure 2 as a running example for explaining Phase 2.

Alattin includes two sub-phases in Phase 2: CFG construction and traversal. In the CFG construction sub-phase, Alattin constructs CFGs for each code example with two kinds of nodes: control $(C T)$ and non-control $(N T)$ nodes. Control nodes represent control-flow statements such as if, while, and for, which control the flow of the program execution. Non-control nodes represent other statements such as method calls or type casts. For example, Statement 5 in the code example (Figure 2) is a control node and Statement 9 is a non-control node. When encountering a control node, say $C T_{i}(i$ indicates the statement id), Alattin also extracts all variables, say $\left\{V_{1}, V_{2}, \ldots, V_{n}\right\}$, that participate in the conditional expression of that node and the condition checks on those variables. For example, the control node $C T_{2}$ includes the $\{$ (val, null-check), (val, instance-check) $\}$ pairs. If the control node includes comparisons with expressions such as method calls, our approach stores those method calls also as additional information within the control node. When encountering a non-control node such as a method call, Alattin extracts variables such as $\{$ receiver, argument $1, \ldots$, arguments $\}$ associated with the method call.

In the CFG traversal sub-phase, Alattin associates gathered condition checks with their related method calls such as Iterator. hasNext. The traversal phase includes two kinds of traversals: backward and forward. Alattin performs a backward traversal from the call site such as $N T_{7}$ of the $F_{i}$ method to collect condition checks on the receiver and argument objects preceding the call site. Similarly, Alattin performs a forward traversal to collect condition checks on the receiver and return objects after the call site of the $F_{i}$ method. In each traversal, Alattin exploits program dependencies for associating condition checks with method calls. Failing to consider these program dependencies may result in programming rules that are not semantically related as shown in the limitations of the PR-Miner [Li and Zhou(2005)] and DynaMine [Livshits and Zimmermann(2005)] approaches. To exploit program dependencies, Alattin uses the concept of dominance with a combination of control-flow and data-flow dependencies.

Definition: A node $N$ dominates another node $M$ in a control flow graph (represented as $N$ dom $M$ ) if every path from the starting node of the CFG to $M$ includes $N$.

Initially, Alattin identifies the dominant $C T_{i}$ nodes for each $N T_{k}$ node. For example, the control node $C T_{6}$ dominates the non-control node $N T_{7}$. Alattin computes the intersection between the variable set associated with the $C T_{i}$ node, say $\left\{V_{1}, V_{2}, \ldots, V_{n}\right\}$, and the receiver or argument variables of the $N T_{k}$ node, say $\{$ receiver, argument $1, \ldots$, arguments $\}$. If the intersection $\left\{V_{1}, V_{2}, \ldots\right.$, $\left.V_{n}\right\} \cap\{$ receiver, argument $1, \ldots$, arguments $\} \neq \emptyset$, Alattin checks whether the $N T_{k}$ node is dependent on the $C T_{i}$ node, i.e., whether there exists at least one variable of $N T_{k}$ node involved in the $C T_{i}$ node and is not redefined in the 
path between $C T_{i}$ and $N T_{k}$ nodes. If the $N T_{k}$ node is dependent on the $C T_{i}$ node, Alattin adds the condition check to the pattern candidate. For example, the extracted condition check for nodes $C T_{6}$ and $N T_{7}$ in the code example is "boolean-check on return of Iterator.hasNext before Iterator.next", which indicates that a boolean-check must be done on the return variable of the hasNext method before the call site of Iterator.next. In our experience, we found that there can be various code examples without any condition checks around an $F_{i}$ method. Failing to consider these code examples can assign incorrect support values to mined patterns. To address this issue, we add an Empty Pattern Candidate to the input database ISD for each such code example.

\subsection{Phase 3: Mining Alternative Patterns}

In Phase 3, Alattin uses our mining algorithms (described in Section 3) to mine patterns in all four pattern formats: And, Or, Xor, and Combo patterns. Alattin applies our mining algorithms on pattern candidates of each $F_{i}$ method individually. The reason is that if we apply mining algorithms on all pattern candidates together, the patterns related to an $F_{i}$ method with a few pattern candidates can be missed due to patterns (related to other $M_{j}$ methods) with a large number of pattern candidates. For each $F_{i}$, Alattin mines patterns in all four pattern formats. We used a min_sup threshold value of 0.4 based on our empirical experience [Thummalapenta and Xie(2009a)].

\subsection{Phase 4: Detecting Neglected Conditions}

In Phase 4, Alattin detects violations of mined patterns in the application under analysis statically. More specifically, Alattin gathers condition checks around each call site of an $F_{i}$ method in the application under analysis. Alattin constructs an itemset $i s_{j}$ using gathered condition checks. For each mined pattern $p c_{k}$ in all patterns of four formats, Alattin uses IssupportedBy (Algorithm 1) to check whether the itemset $i s_{j}$ supports the mined pattern $p c_{k}$. If the itemset does not support the mined pattern, Alattin reports a violation. For each detected violation, Alattin assigns a support value as the same value as the support value of the associated mined pattern used to detect the violation.

\section{Evaluations}

We conducted two evaluations to assess the effectiveness of Alattin. We use the APIs provided by three Java default API libraries to show the existence of alternative patterns. We next use four popular applications to show the benefits and limitations of alternative patterns with respect to false positives and false negatives among detected violations. The details of subjects and results 
of our evaluations are available at https://sites.google.com/site/asergrp/projects/alattin/. We next present research questions addressed in our evaluations.

\subsection{Research Questions}

In our evaluations, we address the following research questions.

- RQ1: How high percentage of And, Or, Xor, and Combo patterns represent real programming rules, respectively? Since real programming rules are required for detecting violations in applications under analysis, this research question helps to show the pattern formats that are suitable for detecting violations.

- RQ2: How low percentage of false negatives and false positives exist among violations detected using And, Or, Xor, and Combo patterns, respectively? Since false positives are one of the common issues faced by existing static defect-detection techniques, this research question helps to show that the patterns that describe nearly complete behavior (such as $\mathrm{Or}$ or Combo) help reduce the number of false positives with no or low increase of false negatives.

\subsection{Subject Applications}

We next present subject applications used in our evaluations. In our evaluations, we used three Java default API libraries and four popular open source libraries. Table 1 shows the characteristics of the subject applications. Columns "Classes" and "Methods" show the number of classes and methods, respectively. For mining patterns of three Java default API libraries, we gathered 49858, 5555, and 15052 code examples for Java Util, Java Transaction, and Java SQL, respectively. Column "KLOC" shows the kilo lines of code in each subject application.

The Java Util package ${ }^{2}$ includes the collections framework and other popular utilities used by many different applications. Java Transactions ${ }^{3}$ and Java $\mathrm{SQL}^{4}$ are industry standards for developing multi-tier server-side Java applications. Hibernate $\mathrm{e}^{5}$ and $\mathrm{HsqlDB}^{6}$ abstract relational databases to use an object-oriented methodology. Columba ${ }^{7}$ is an open source email-client application written in Java. Columba provides a user-friendly graphical interface

\footnotetext{
2 http://java.sun.com/j2se/1.4.2/docs/api/java/util/package-summary. html

3 http://java.sun.com/javaee/technologies/jta/

4 http://java.sun.com/j2se/1.4.2/docs/api/java/sql/package-summary. html

5 http://www.hibernate.org/

6 http://hsqldb.org/

7 http://sourceforge. net/projects/columba/
} 
Table 1 Subject applications and their characteristics.

\begin{tabular}{|l|c|c|c|}
\hline Application & \#Classes & \#Methods & KLOC \\
\hline Java Util APIs & 19 & 144 & - \\
\hline Java Transaction APIs & 7 & 37 & - \\
\hline Java SQL APIs & 14 & 93 & - \\
\hline Hibernate & 478 & 4334 & 118 \\
\hline HsqlDB & 143 & 1178 & 41 \\
\hline Columba & 1500 & 7674 & 136 \\
\hline BCEL & 357 & 2691 & 32 \\
\hline Total & 2518 & 16151 & 327 \\
\hline
\end{tabular}

Table 2 Alternative patterns mined by Alattin

\begin{tabular}{|l|c|c|c|c|c|c|c|c|c|}
\hline \multirow{2}{*}{ Application } & \multicolumn{3}{|c|}{ And Patterns } & \multicolumn{5}{c|}{ Or Patterns } \\
\cline { 2 - 9 } & Total & \#RR & \#PR & \#FP & Total & \#RR & \#PR & \#FP \\
\hline Java Util & 40 & 34 & 0 & 6 & 51 & 25 & 19 & 7 \\
\hline Java Sql & 26 & 26 & 0 & 0 & 24 & 21 & 3 & 0 \\
\hline $\begin{array}{l}\text { Java } \\
\text { Transaction }\end{array}$ & 3 & 3 & 0 & 0 & 9 & 2 & 4 & 3 \\
\hline & \multicolumn{3}{|c|}{ Xor Patterns } & \multicolumn{5}{c|}{ Combo Patterns } \\
\cline { 2 - 10 } & Total & \#RR & \#PR & \#FP & Total & \#RR & \#PR & \#FP \\
\hline Java Util & 54 & 35 & 11 & 8 & 50 & 32 & 11 & 7 \\
\hline Java Sql & 33 & 30 & 3 & 0 & 24 & 21 & 3 & 0 \\
\hline $\begin{array}{l}\text { Java } \\
\text { Transaction }\end{array}$ & 8 & 2 & 4 & 2 & 8 & 2 & 4 & 2 \\
\hline
\end{tabular}

RR: Real Rules, PR: Partial Rules, FP: False Positives

and is suitable for internationalization support. The BCEL library ${ }^{8}$, developed by Apache, is mainly used to analyze, create, and manipulate Java class files. We selected these applications, since these applications are popular open source applications and are used as subjects in evaluating previous related approaches [Weimer and Necula(2005), Thummalapenta and Xie(2009b)].

\subsection{RQ1: Alternative Patterns}

We next address the first research question of whether alternative patterns exist in real applications and how high percentage of those patterns represent real programming rules. To address this question, we configured Alattin, which by default accepts an application under analysis and mines patterns for thirdparty APIs, to accept a set of classes and methods directly. In this mode of operation, Alattin mines patterns (programming rules) for the APIs of the given classes and methods.

Table 2 shows the patterns mined in all four formats: And, Or, Xor, and Combo. For each pattern format, Columns "Total", "RR", "PR", and "FP" show the total number of mined patterns, real rules, partial rules, and false positives, respectively. Real rules describe properties that must be satisfied when using an API method. In real rules, all alternatives are real properties.

8 http://jakarta.apache.org/bcel/ 


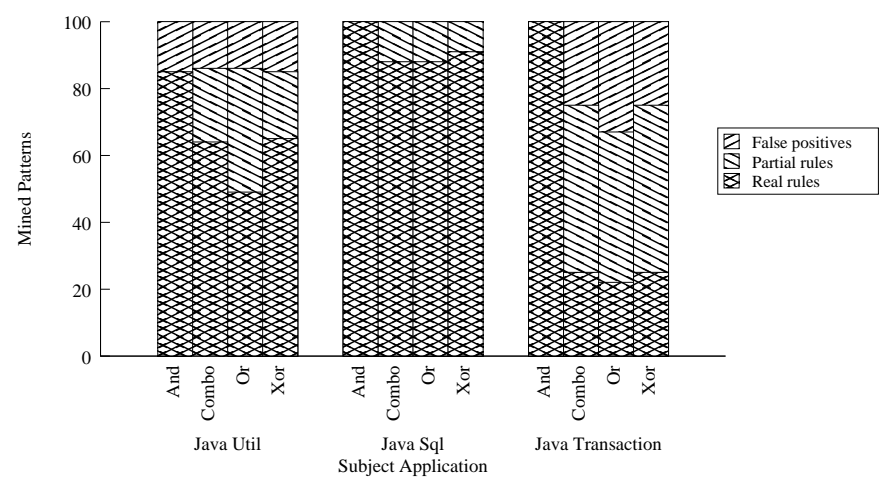

Fig. 9 Classification of mined patterns

In contrast to real rules, some alternatives in Partial rules do not represent real properties. The reason for introducing partial rules is that partial rules are as effective as real rules in reducing false-positive defects; however, partial rules can increase false-negative defects due to false-positive alternatives among mined patterns. False positives represent mined patterns where none of the alternatives represents real properties. To mine patterns in all four pattern formats, Alattin took 13, 1, and 1 seconds for Java Util, Java Sql, and Java Transaction, respectively. All experiments were conducted on a machine with $2.2 \mathrm{GHz}$ Intel processor and 3GB RAM. We used available on-line documentations, JML specifications ${ }^{9}$, or source code of applications for classifying mined patterns into these three categories.

Figure 9 shows the percentages of real, partial, and false-positive rules among mined patterns of each pattern format. In summary, a high percentage of And, Or, Xor, and Combo patterns represent real rules. We also found that $O r, X o r$, and Combo patterns include new real rules that do not exist among And patterns. Since existing approaches mine only And patterns, our results show that new defects can be detected using Or, Xor, and Combo patterns. The figure also shows that, except And patterns, all other pattern formats include a considerable percentage of partial rules. Therefore, although these three pattern formats can help reduce false positives, these three pattern formats can result in false negatives among detected violations. Furthermore, Or patterns have higher percentage of partial rules compared to Xor and Combo patterns, indicating that $O r$ patterns result in more false negatives compared to Xor and Combo patterns.

We next present example patterns in each pattern format for the read method of the java.util. jar. JarInputstream class, which extends the zipInputstream class. This class is used for reading the contents of a Jar file from any input stream such as FileInputstream. This class includes three methods: get NextEntry, getNextJarEntry, and read. The getNextEntry method reads the next Zip file entry, represented as an instance of the zipEntry class, and positions the stream

9 http: / / www . eecs.ucf.edu/ leavens/JML/ 


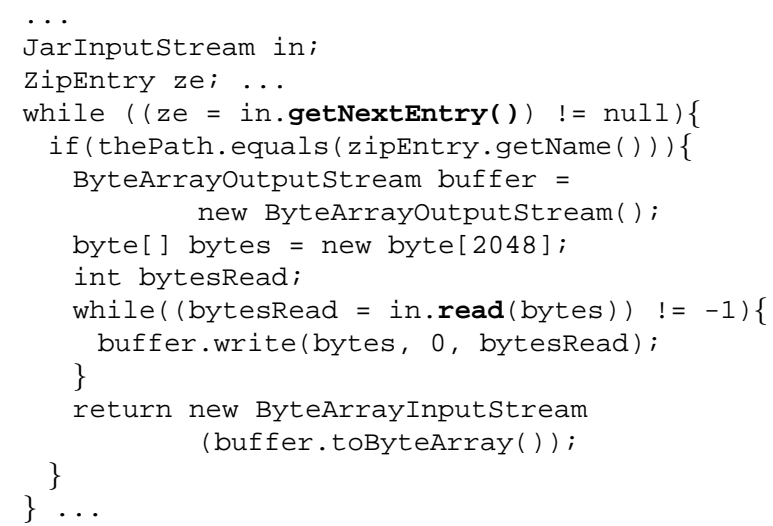

Fig. 10 Alternative patterns mined for the read method of the JarInputStream class.

at the beginning of the entry data in the Jar file. On the other hand, the getNextJarEntry method reads the next Jar file entry, represented as an instance of the JarEntry class, and positions the stream at the beginning of the entry data. Indeed, the JarEntry class extends the zipEntry class and includes additional methods such as getAttributes for reading the attributes specific to the Jar file. In general, programmers use either getNextentry or getNextJarEntry for iterating through the entries in the Jar file and for reading the contents using the read method. Furthermore, if there is only one entry to read from the Jar file, the read method is used directly without using either getNextEntry or getNextJarEntry. Figure 10 shows an example usage of getNextEntry and read methods. This code example is extracted from Apache's Jakarta Cactus project ${ }^{10}$.

Figure 11 shows the patterns mined for the read method in all four formats. The And pattern includes only one alternative $P_{1}$, which describes that there should be a condition check with "- 1 " on the return value of the read method. Here, "- 1 " indicates that the end of the entry is reached. The Or Pattern includes two alternatives " $P_{1} \vee P_{2}$ ", where $P_{2}$ indicates that there should be a null-check on the return of the getNextJarEntry method. The reason that the And pattern could not mine the alternative $P_{2}$ is that there are various scenarios where $P_{1}$ and $P_{2}$ are not used together. For example, when $P_{2}$ is used, programmers often get the size of the buffer to be read using the getsize method of JarEntry, which is the return type of the getNextJarEntry method. Therefore, programmers often do not explicitly check the return value of the read method, when the getNextJarEntry method is used. Figure 11 also shows that there are two Xor patterns. Interestingly, the second pattern " $P_{2} \oplus P_{3}$ " shows that programmers often use either getNextJarEntry or getNextEntry, but not both together, since the related pattern " $P_{2} \vee P_{3}$ " is not being mined. However, the Xor pattern alone could not mine the relation among all alter-

10 http://jakarta.apache.org/cactus/ 


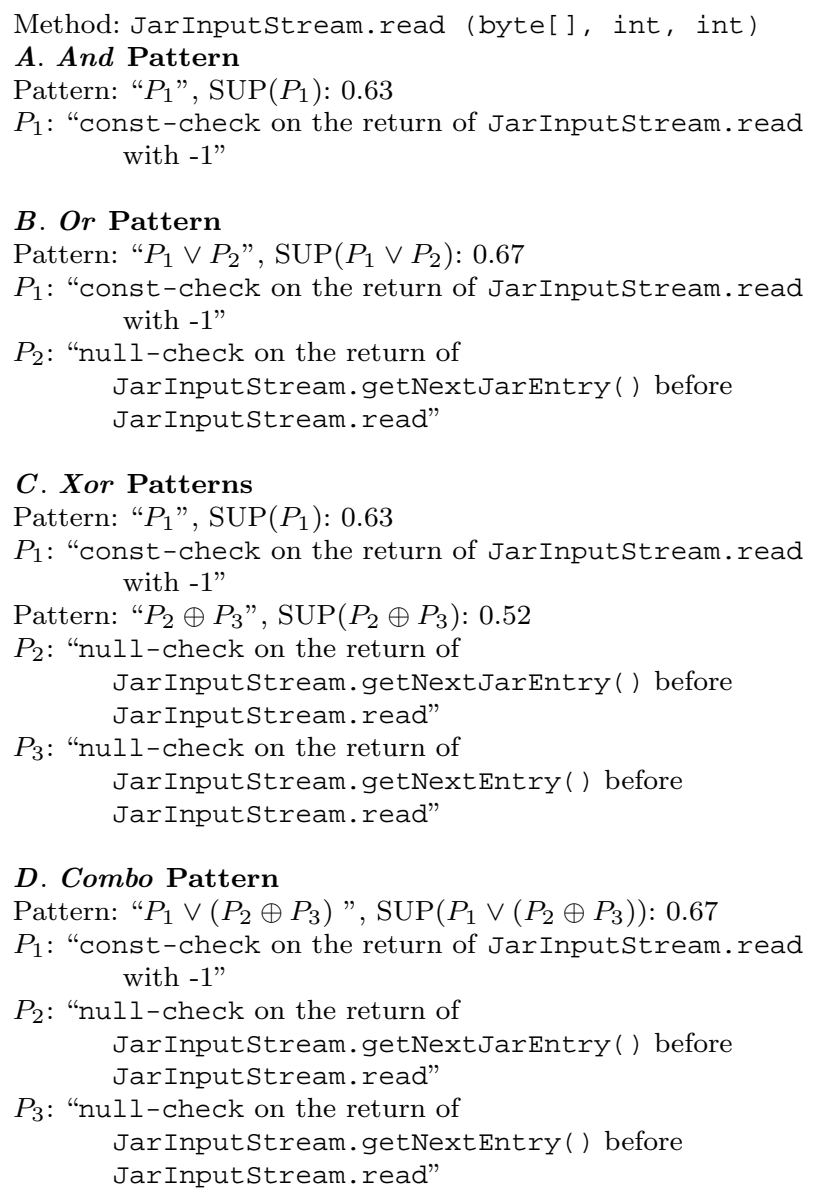

Fig. 11 Alternative patterns mined for the read method of the JarInputStream class.

natives $P_{1}, P_{2}$, and $P_{3}$. The Combo pattern addresses this issue via mining the pattern " $P_{1} \vee\left(P_{2} \oplus P_{3}\right)$ ", and shows the relation among all alternatives.

\subsection{RQ2: False Positives and False Negatives}

We next address the second research question of whether alternative patterns help reduce false positives among detected violations. We also address whether these patterns introduce no or a low percentage of false negatives among detected violations. To address this question, we used the four subject applications (Hibernate, Columba, BCEL, and HsqlDB) shown in Table 1. In particular, we mined patterns in all four formats from these applications under analysis and apply mined patterns on those applications to detect violations. We next inspected detected violations to classify violations as real defects or false positives based on available specifications such as JML and call sites of 
Table 3 Analysis of violations detected in subject applications.

\begin{tabular}{|c|c|c|c|c|c|c|c|c|c|c|c|c|c|}
\hline \multirow{2}{*}{ Application } & \multirow{2}{*}{\begin{tabular}{|l|} 
\# Real \\
Defects
\end{tabular}} & \multicolumn{6}{|c|}{ And Patterns } & \multicolumn{6}{|c|}{ Or Patterns } \\
\hline & & Total & \#RD & \#FN & $\%$ & \#FP & $\%$ & Total & \#RD & \#FN & $\%$ & \#FP & $\%$ \\
\hline Columba & 49 & 117 & 26 & 23 & 47 & 91 & 78 & 113 & 41 & 8 & 16.3 & 72 & 63.7 \\
\hline Hibernate & 22 & 93 & 14 & 8 & 36 & 71 & 76 & 177 & 17 & 5 & 22.7 & 160 & 90.4 \\
\hline Hsqldb & 6 & 13 & 6 & 0 & 0 & 7 & 53.8 & 5 & 5 & 1 & 16.7 & 0 & 0 \\
\hline \multirow{3}{*}{ BCEL } & 1 & 2 & 0 & 1 & 100 & 2 & 100 & 13 & 1 & 0 & 0 & 12 & 92.3 \\
\hline & & \multicolumn{6}{|c|}{ Xor Patterns } & \multicolumn{6}{|c|}{ Combo Patterns } \\
\hline & & Total & \#RD & $\# \mathrm{FN}$ & $\%$ & \#FP & $\%$ & Total & \#RD & \#FN & $\%$ & $\# \mathrm{FP}$ & $\%$ \\
\hline Columba & 49 & \begin{tabular}{|l|}
164 \\
\end{tabular} & 49 & 0 & 0 & 115 & 73 & 144 & 47 & \begin{tabular}{|l|}
2 \\
\end{tabular} & 4 & 97 & 67 \\
\hline Hibernate & 22 & 214 & 21 & 1 & 4.5 & 193 & 90.2 & 195 & 19 & 3 & $\mid 13.6$ & \begin{tabular}{|l|}
176 \\
\end{tabular} & 90.3 \\
\hline HsqlDB & 6 & 11 & 6 & 0 & 0 & 5 & 45.5 & 10 & 6 & 0 & 0 & 4 & 40 \\
\hline BCEL & 1 & 20 & 1 & 0 & 0 & 19 & 95 & 16 & 1 & 0 & 0 & 15 & 93.8 \\
\hline
\end{tabular}

RD: Real Defects, FN: False Negatives, FP: False Positives

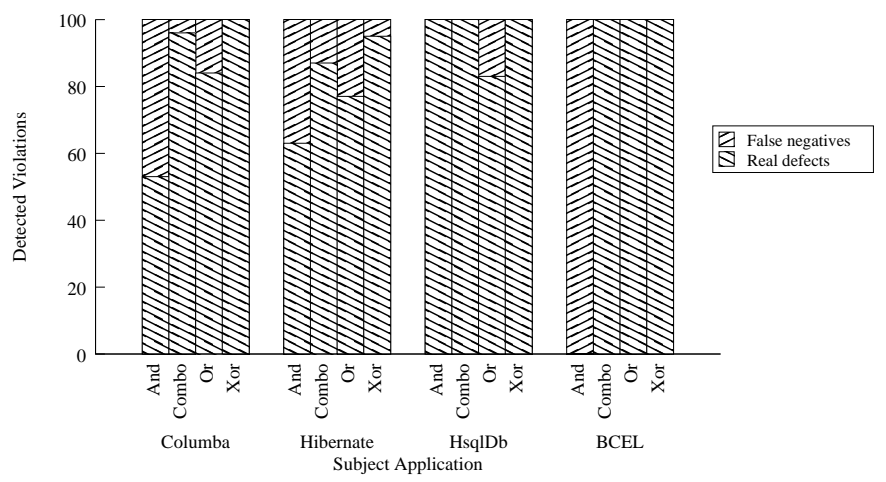

Fig. 12 Real defects and false negatives among detected violations.

related API methods in source code of these subject applications. In our inspection, we ignored the violations related to API methods whose all pattern formats include only one alternative, since our objective is to show the benefits and limitations of alternative patterns. The primary reason is that such patterns do not help show benefits of alternative patterns, since those patterns have the same number of false positives or false negatives in all pattern formats. To compute false negatives, we need a baseline that shows the number of defects exist in subject applications. Since such a baseline does not exist for these applications, we identified all distinct real defects detected using patterns in all pattern formats and used those defects as a baseline for computing false negatives among violations detected using each pattern format.

Table 3 shows detected violations in all subject applications. Column "Real Defects" shows the total number of distinct defects detected using all pattern formats in each application. We used these defects as a baseline for computing the number of false negatives among violations detected using each pattern format. For each pattern format, Columns "Total", "RD", "FN", and "FP" show the total number of violations, real defects, false negatives (their percentage), and false positives (their percentage), respectively. We next summarize our 


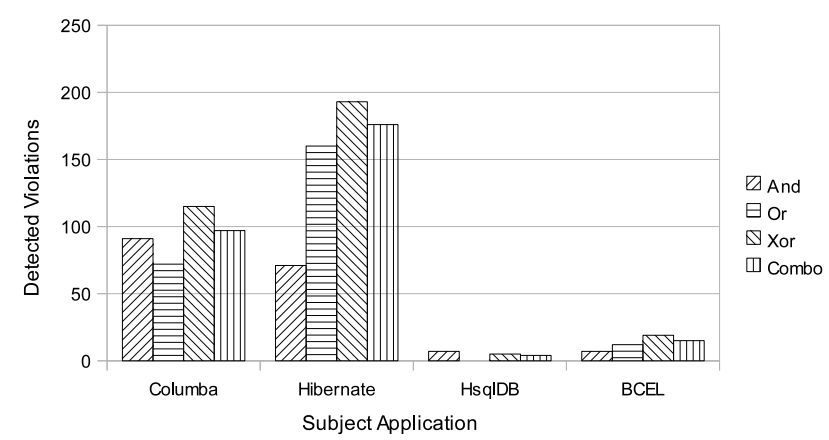

Fig. 13 False positives among detected violations.

findings for each pattern format with respect to real defects, false negatives, and false positives.

Real defects and False negatives. Figure 12 shows comparison between real defects and false negatives for the four pattern formats in each subject application. The figure shows that Or, Xor, and Combo patterns helped detect new defects that are not detected using And patterns. For example, in the Columba application, Or patterns detected 41 real defects, whereas And patterns detected only 26 real defects. The reason for new defects is due to the new patterns mined using the Or, Xor, and Combo pattern formats.

Regarding false negatives, our results show that the violations detected using And patterns include a high percentage of false negatives in 3 out of 4 applications. Since And patterns represent patterns that can be mined by existing approaches, the results show the ineffectiveness of existing approaches in detecting defects in applications under analysis. Among Or, Xor, and Combo patterns, violations detected using $O r$ patterns have a higher number of false negatives compared to the violations detected using Xor and Combo patterns. For example, in the Columba application, violations detected using Or patterns include $8(16.3 \%)$ false negatives compared to $0(0 \%)$ and $2(4 \%)$ false negatives among violations detected using Xor and Combo patterns, respectively. The primary reason is that $O r$ patterns often include partial rules (as shown in Figure 9), resulting in false negatives among detected violations. The results show that Xor patterns are quite effective in detecting defects compared to all three other pattern formats: And, Or, and Combo. However, Combo patterns are also shown to be effective than $O r$ patterns and have similar effectiveness as that of Xor patterns.

False positives. Figure 13 shows the number of false positives among violations detected using patterns in each pattern format. Initially, we expected that $O r$ and Combo patterns help reduce a high percentage of false positives among violations detected using And and Xor patterns. However, contrary to our expectation, the number of false positives is high among violations detected using $O r$ and Combo patterns. For example, in the Hibernate application, the numbers of false positives are 71, 160, 193, and 176 among violations detected using And, Or, Xor, and Combo patterns, respectively. 


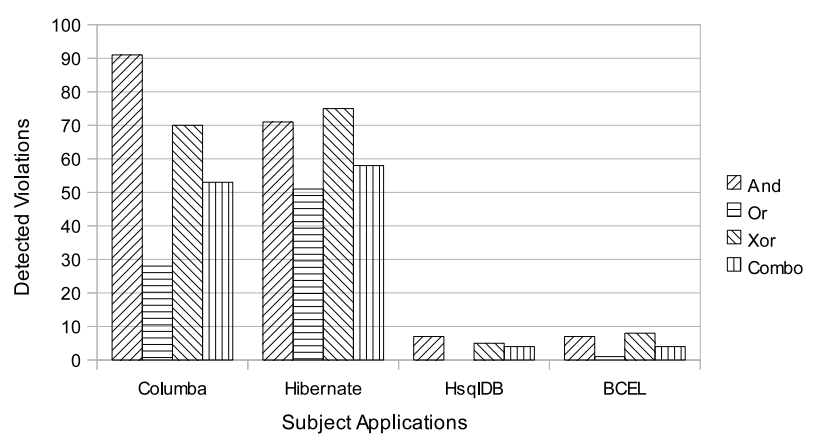

Fig. 14 False positives among detected violations related to API methods with And patterns.

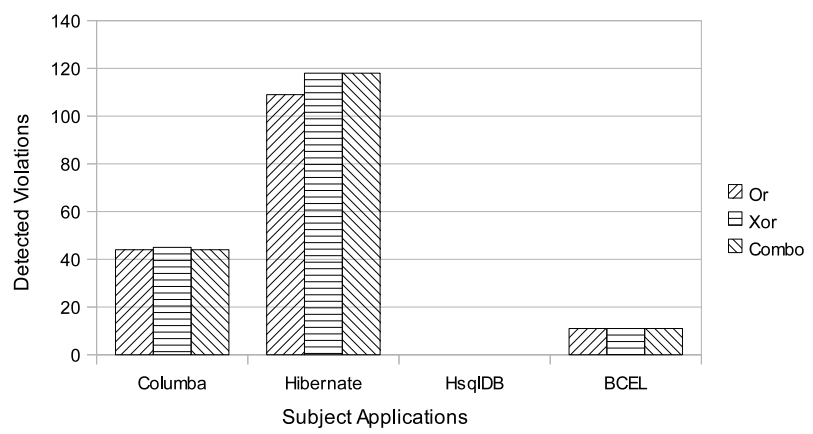

Fig. 15 False positives among detected violations related to API methods without And patterns.

In our manual analysis of these false positives, we identified an interesting phenomenon: the majority of false positives is related to the API methods that do not have any patterns mined using the And pattern format and have new patterns mined using one or more of the Or, Xor, and Combo pattern formats. To illustrate this scenario, we classified all false positives among detected violations into two categories: FPAnd and FPWithOutAnd. FPAnd includes all false positives detected using patterns (Or, Xor, and Combo patterns) related to API methods that have mined patterns using the And pattern format. In contrast, FPWithOutAnd includes all false positives detected using patterns (Or, Xor, and Combo patterns) related to API methods that do not have mined patterns using the And pattern format. Figures 14 and 15 show the classification of false positives for categories FPAnd and FPWithOutAnd, respectively. Figure 14 shows that $O r$ patterns help significantly reduce the number of false positives among detected violations compared to And and Xor patterns. Although Combo patterns help reduce false positives, these patterns are not as effective as $O r$ patterns. The primary reason is that most of the Combo patterns are similar to Xor patterns based on our algorithm described in Section 3. 
Figure 15 shows the classification of false positives for API methods without And patterns. As shown in the figure, neither Or nor Combo patterns help reduce false positives among violations detected using Xor patterns. The primary reason is that most of these mined patterns are false positives, resulting in false positives among their detected violations. We next summarize our findings.

\subsection{Summary}

In summary, based on our results, comparing to Or, Xor, and Combo patterns, And patterns are not effective in detecting defects and result in both false positives and false negatives among detected violations. Although Xor patterns are effective in detecting defects, these patterns result in a large number of false positives among detected violations. On the other hand, Or patterns are effective in reducing false positives, but, result in false negatives as shown in our results. Combo patterns can perform reasonably well with respect to both false positives and false negatives. However, Or or Combo patterns often result in false-positive patterns for those API methods without any And patterns. Therefore, based on our empirical results, the best pattern-mining approach (in terms of reducing both false positives and false negatives) is to first mine And patterns for API methods and next mine Combo patterns. Among violations detected using Combo patterns, the best violation-detection approach is to assign higher priority to the violations of API methods with And patterns compared to the violations of API methods without And patterns. The primary reason is that the former violations are more likely to be real defects compared to the latter violations.

\section{Threats to Validity}

The threats to external validity primarily include the degree to which the subject programs and used CSE are representative of true practice. The current subjects range from small-scale libraries such as Java SQL APIs to large-scale libraries such as BCEL and Hibernate. We used only one CSE, i.e., Google code search, which is a well-known CSE. These threats could be reduced by more experiments on wider types of subjects and by using other CSEs in future work. The threats to internal validity are instrumentation effects that can bias our results. Faults in our Alattin prototype might cause such effects. There can be errors in our inspection of source code for confirming rules or defects. To reduce these threats, we inspected available specifications and also call sites in source code. 


\section{Discussion}

Since the inception of the Mining Software Engineering Data methodology, researchers have explored the mining of various pattern types ranging from simple itemsets that represent necessary condition checks around API method calls [Thummalapenta and Xie(2009a)] to complex graph-based pattern types that describe the usage patterns of one or multiple objects [Nguyen et al(2009)Nguyen, Nguyen, Pham, Al-Kofahi, and Nguyen]. In this paper, we focused on mining itemset pattern types in four pattern formats and showed their benefits and limitations. In our future work, we plan to explore other pattern types such as sequences [Srikant and Agrawal(1996)] and graph-based pattern types [Han and Kamber(2000)] to develop new mining algorithms for mining those pattern types in various formats and show their benefits and limitations in defect detection.

In this paper, we proposed a greedy technique for mining patterns in $O r$, Xor, and Combo pattern formats. Our greedy technique (shown as the function ApplyGreedy in Algorithm 2) chooses one parent pattern candidate with the highest support value for each pattern candidate. The primary reason for adopting the greedy technique is to reduce the search space of pattern candidates. In future work, we plan to explore other techniques such as clustering techniques [Han and Kamber(2000)]. In particular, we plan to first group items (among itemsets in the input database) that are closely related to each other. We next apply our mining algorithms without the greedy technique on each cluster individually. We expect that the number of items in each cluster could be low and help reduce the search space of pattern candidates significantly. We also plan to adopt some properties used by Zhao et al. [Zhao et al(2006)Zhao, Zaki, and Ramakrishnan] for handling search space of pattern candidates while mining patterns.

Our current implementation sometimes is not precise and cannot identify equivalent but syntactically different conditions. For example, our current implementation considers the conditions $a>0$ and $a \geq 1$ as different. In future work, we plan to address these issues using more precise static analysis that can identify equivalent conditions. Furthermore, we use intra-procedural analysis for detecting violations. Therefore, in a few cases, some detected defects could be false positives with respect to the entire system point of view. However, this limitation does not affect the results of comparing the benefits and limitations of our four pattern formats, since we use the same analysis for all four pattern formats. In future work, we plan to address this limitation by using inter-procedural analysis.

\section{Related Work}

PR-Miner developed by Li and Zhou [Li and Zhou(2005)] uses frequent itemset mining to mine programming rules from $\mathrm{C}$ code and detect their violations. DynaMine developed by Livshits and Zimmermann [Livshits and Zimmer- 
mann(2005)] uses association rule mining to extract simple rules from software revision histories for Java code and detect defects related to rule violations. PR-Miner or DynaMine may suffer from issues of a high number of false positives since their rule elements are not necessarily associated with program dependencies. Furthermore, these approaches target at only frequent patterns, whereas Alattin can mine alternative patterns that include both frequent and infrequent alternatives.

Another related approach to our Alattin approach is the approach developed by Chang et al. [Chang et al(2007)Chang, Podgurski, and Yang] that applies frequent subgraph mining on $\mathrm{C}$ code to mine condition rules and to detect neglected conditions. Both Alattin and their approach target at the same type of defects: neglected conditions. Alattin significantly differs from Chang et al.'s approach in three main aspects. First, their approach cannot mine infrequent alternatives. Second, their approach is limited on a much smaller scale of code repositories (in fact, only one project code base) than Alattin, which exploits a CSE to search for relevant code examples from open source code available on the web. Third, the scalability of their approach is heavily limited by its underlying graph mining algorithms, which are known to suffer from scalability issues. In contrast, Alattin uses our new ImMiner algorithm based on frequent itemset mining, being much more scalable.

Williams and Hollingsworth [Williams and Hollingsworth(2005)] incorporate an API call return value checker for $\mathrm{C}$ code, which checks that a value returned by an API call is checked before being used. This type of return-value checking before use falls into a subset of the types of rules being mined by Alattin. Different from their tool, Alattin does not require or rely on version histories, which may not include the types of defect fixing (required by their tool) related to the rules being mined. Acharya et al. [Acharya et al(2006)Acharya, $\mathrm{Xie}$, and $\mathrm{Xu}$ ] developed a tool to mine interface details (such as an API call's return values on success or failure and error flags) from model-checker traces for $\mathrm{C}$ code, and then mine interface robustness properties for defect detection. Similar to the tool of Williams and Hollingsworth [Williams and Hollingsworth(2005)], Acharya et al.'s tool mines only a subset of neglected conditions (e.g., return-value checking before use) mined by Alattin. In addition, as shown by Acharya et al. [Acharya et al(2006)Acharya, Xie, and Xu], only the interface details of 22 out of 60 POSIX API functions can be successfully mined by their tool, whereas Alattin exploits a CSE to alleviate the issue by collecting relevant API call usages from the web. Furthermore, these approaches cannot mine alternative patterns targeted by Alattin.

Engler et al. [Engler et al(2001)Engler, Chen, Hallem, Chou, and Chelf] proposed a general approach for detecting defects in $\mathrm{C}$ code by applying statistical analysis to rank deviations from programmer beliefs inferred from source code. Their approach allows users to define rule templates, which are not required by our approach. In addition, their approach also cannot mine infrequent alternatives targeted by our Alattin approach.

Although ours is the first approach to propose new pattern formats such as disjunctive and exclusive-disjunctive pattern formats for mining software 
engineering data, a few approaches have been proposed (in the data mining research area) that target at mining patterns in these preceding formats for other applications such as market basket analysis [Agrawal et al(1996)Agrawal, Mannila, Srikant, Toivonen, and Verkamo]. Zhao et al. [Zhao et al(2006)Zhao, Zaki, and Ramakrishnan] proposed an approach, called BLOSOM, that targets at mining itemset patterns in four pattern formats: conjunctive, disjunctive, conjunction of disjunctive, and disjunction of conjunctive. In contrast to their BLOSOM approach, our Alattin approach additionally proposes the exclusive-disjunctive pattern format and includes a greedy technique for handling the search space of pattern candidates. In future work, we plan to adopt some properties used by their approach for handling the search space of pattern candidates while mining disjunctive patterns. Nanavati et al. [Nanavati et al(2001)Nanavati, Chitrapura, Joshi, and Krishnapuram] proposed an approach for mining disjunctive association rules. Given a minimum confidence min_conf, their approach uses concepts from propositional logic for pruning the association rules that do not have confidence higher than min_conf. Shimizu and Miura [Shimizu and Miura(2005)] proposed algorithms for mining disjunctive sequence patterns. In contrast to these two preceding approaches, our Alattin approach targets at mining itemset patterns in disjunctive and exclusive-disjunctive pattern formats.

Finally, our previous approaches PARSEWeb [Thummalapenta and Xie(2007)] and CAR-Miner [Thummalapenta and Xie(2009b)] also exploit code search engines for gathering relevant code samples. PARSEWeb accepts queries of the form "Source $\rightarrow$ Destination" and mines frequent method-invocation sequences that accept Source and produce Destination. Although Alattin uses code search engines for gathering relevant code examples, Alattin targets at mining patterns that describe programming rules that should be obeyed while reusing APIs. Unlike PARSEWeb, which mines frequent sequences, Alattin mines alternative patterns with both frequent and infrequent alternatives. CAR-Miner also incorporates a new mining algorithm for mining exceptionhandling rules in the form of sequence association rules. CAR-Miner and Alattin differ significantly in three major aspects. (1) CAR-Miner mines rules for detecting exception-handling-related defects, whereas Alattin mines rules for detecting neglected conditions. (2) Alattin is a more general approach compared to CAR-Miner and can be applied to enhance various existing miningbased approaches including CAR-Miner for detecting alternative rules. (3) CAR-Miner mines new kinds of patterns for reducing false negatives (i.e., detecting new kinds of exception-handling defects). In contrast, Alattin mines new kinds of patterns for reducing false positives.

\section{Conclusion}

To reduce false positives in static defect detection based on code mining, we have developed a novel approach, called Alattin, that includes new mining algorithms and a technique for detecting neglected conditions. Our new mining 
algorithms mine patterns in four pattern formats: And, Or, Xor, and Combo. In our evaluations, we show the benefits and limitations of these pattern formats with respect to false positives and false negatives among detected violations that represent neglected conditions in applications under analysis. Our evaluation results show that the best pattern-mining approach (in terms of reducing both false positives and false negatives) is to first mine And patterns for API methods and next mine Combo patterns. Among violations detected by Combo patterns, the best violation-detection approach is to assign higher priority to the violations of API methods with And patterns compared to the violations of API methods without And patterns. The primary reason is that the former violations are more likely to be real defects compared to the latter violations.

In this paper, we follow a problem-driven methodology in advancing the field of mining software engineering data. Our current approach and previous approach [Thummalapenta and Xie(2009b)] serve as examples in this direction. More specifically, in our approaches, we empirically investigate problems in the software engineering domain and identify required types of patterns for addressing those problems. We further develop new mining algorithms for mining these required types of patterns, rather than being constrained by available mining algorithms from the data mining community. Our approaches primarily target at reducing false negatives and false positives among detected violations. Our previous approach [Thummalapenta and Xie(2009b)], which mines programming rules as sequence association rules, focuses on reducing false negatives by detecting new kinds of defects. In contrast, our current approach focuses on a new sub-direction of reducing false positives among detected violations. In future work, we plan to further expand our research by investigating broader types of problems, patterns, mining algorithms, and defects.

Acknowledgements This work is supported in part by NSF grant CCF-0725190, ARO grant W911NF-08-1-0443, and ARO grant W911NF-08-1-0105 managed by NCSU Secure Open Systems Initiative (SOSI).

\section{References}

[Acharya et al(2006)Acharya, Xie, and Xu] Acharya M, Xie T, Xu J (2006) Mining interface specifications for generating checkable robustness properties. In: Proc. ISSRE, pp $311-320$

[Acharya et al(2007)Acharya, Xie, Pei, and Xu] Acharya M, Xie T, Pei J, Xu J (2007) Mining API patterns as partial orders from source code: From usage scenarios to specifications. In: Proc. ESEC/FSE, pp 25-34

[Agrawal et al(1996)Agrawal, Mannila, Srikant, Toivonen, and Verkamo] Agrawal R, Mannila H, Srikant R, Toivonen H, Verkamo AI (1996) Fast discovery of association rules. In: Advances in knowledge discovery and data mining, pp 307-328

[Ammons et al(2002)Ammons, Bodik, and Larus] Ammons G, Bodik R, Larus JR (2002) Mining specifications. In: Proc. POPL, pp 4-16

[Burdick et al(2001)Burdick, Calimlim, and Gehrke] Burdick D, Calimlim M, Gehrke J (2001) MAFIA: A maximal frequent itemset algorithm for transactional databases. In: Proc. ICDE, pp 443-452 
[Chang et al(2007)Chang, Podgurski, and Yang] Chang RY, Podgurski A, Yang J (2007) Finding what's not there: a new approach to revealing neglected conditions in software. In: Proc. ISSTA, pp 163-173

[DMSE(2010)] DMSE (2010) Bibliography on mining software engineering data. https://sites.google.com/site/asergrp/dmse/

[Engler et al(2001)Engler, Chen, Hallem, Chou, and Chelf] Engler D, Chen DY, Hallem S, Chou A, Chelf B (2001) Bugs as deviant behavior: a general approach to inferring errors in systems code. In: Proc. SOSP, pp 57-72

[Ernst et al(2001)Ernst, Cockrell, Griswold, and Notkin] Ernst M, Cockrell J, Griswold W, Notkin D (2001) Dynamically discovering likely program invariants to support program evolution. IEEE Trans Softw Eng 27(2):99-123

[GCSE(2006)] GCSE (2006) Google code search engine. http://www.google.com/codesearch

[Han and Kamber(2000)] Han J, Kamber M (2000) Data mining: concepts and techniques. Morgan Kaufmann Publishers Inc.

[KODERS(2005)] KODERS (2005) The Koders source code search engine. http: // www . koders. com

[Lethbridge et al(2003)Lethbridge, Singer, and Forward] Lethbridge T, Singer J, Forward A (2003) How software engineers use documentation: The state of the practice. In: IEEE Software, pp 35-39

[Li and Zhou(2005)] Li Z, Zhou Y (2005) PR-Miner: Automatically extracting implicit programming rules and detecting violations in large software codes. In: Proc. FSE, pp $306-315$

[Livshits and Zimmermann(2005)] Livshits VB, Zimmermann T (2005) Dynamine: Finding common error patterns by mining software revision histories. In: Proc. ESEC/FSE, pp 296-305

[Nanavati et al(2001)Nanavati, Chitrapura, Joshi, and Krishnapuram] Nanavati AA, Chitrapura KP, Joshi S, Krishnapuram R (2001) Mining generalised disjunctive association rules. In: Proc. CIKM, pp 482-489

[Nguyen et al(2009)Nguyen, Nguyen, Pham, Al-Kofahi, and Nguyen] Nguyen TT, Nguyen HA, Pham NH, Al-Kofahi JM, Nguyen TN (2009) Graph-based mining of multiple object usage patterns. In: Proc. ESEC/FSE, pp 383-392

[Ramanathan et al(2007)Ramanathan, Grama, and Jagannathan] Ramanathan MK, Grama A, Jagannathan S (2007) Path-sensitive inference of function precedence protocols. In: Proc. ICSE, pp 240-250

[Shimizu and Miura(2005)] Shimizu K, Miura T (2005) Disjunctive sequential patterns on single data sequence and its anti-monotonicity. In: Proc. MLDM, pp 376-383

[Shoham et al(2007)Shoham, Yahav, Fink, and Pistoia] Shoham S, Yahav E, Fink S, Pistoia M (2007) Static specification mining using automata-based abstractions. In: Proc. ISSTA, pp 174-184

[Srikant and Agrawal(1996)] Srikant R, Agrawal R (1996) Mining sequential patterns: Generalizations and performance improvements. In: Proc. EDBT, pp 3-17

[Thummalapenta and Xie(2007)] Thummalapenta S, Xie T (2007) PARSEWeb: A programmer assistant for reusing open source code on the web. In: Proc. ASE, pp 204-213

[Thummalapenta and Xie(2009a)] Thummalapenta S, Xie T (2009a) Alattin: Mining alternative patterns for detecting neglected conditions. In: Proc. ASE, pp 283-294

[Thummalapenta and Xie(2009b)] Thummalapenta S, Xie T (2009b) Mining exceptionhandling rules as sequence association rules. In: Proc. ICSE, pp 496-506

[Wasylkowski et al(2007)Wasylkowski, Zeller, and Lindig] Wasylkowski A, Zeller A, Lindig C (2007) Detecting object usage anomalies. In: Proc. ESEC/FSE, pp 35-44

[Weimer and Necula(2005)] Weimer W, Necula G (2005) Mining temporal specifications for error detection. In: Proc. TACAS, pp 461-476

[Williams and Hollingsworth(2005)] Williams CC, Hollingsworth JK (2005) Recovering system specific rules from software repositories. In: Proc. MSR, pp 1-5

[Yang et al(2006)Yang, Evans, Bhardwaj, Bhat, and Das] Yang J, Evans D, Bhardwaj D, Bhat T, Das M (2006) Perracotta: mining temporal API rules from imperfect traces. In: Proc. ICSE, pp 282-291

[Zhao et al(2006)Zhao, Zaki, and Ramakrishnan] Zhao L, Zaki MJ, Ramakrishnan N (2006) BLOSOM: a framework for mining arbitrary boolean expressions. In: Proc. KDD, pp $827-832$ 